\title{
Atmospheric monitoring using the Cherenkov Transparency Coefficient for the Cherenkov Telescope Array
}

\author{
Stanislav Stefanik ${ }^{1, *}$ and Dalibor Nosek ${ }^{1}$ for the CTA Consortium** \\ ${ }^{1}$ Institute of Particle and Nuclear Physics, Faculty of Mathematics and Physics, \\ Charles University, V Holesovickach 2, 18000 Prague 8, Czech Republic
}

\begin{abstract}
The future ground-based gamma-ray observatory, the Cherenkov Telescope Array (CTA) will require reliable monitoring of the atmosphere which is an inherent part of the detector. We discuss here the implementation of the extended method of the Cherenkov Transparency Coefficient for the atmospheric calibration for the CTA. The method estimates the atmospheric transmission of Cherenkov light, relying on the measurement of the rates of cosmic ray-induced air showers that trigger different pairs of telescopes. We examine the performance of our approach utilizing Monte Carlo simulations assuming various atmospheric conditions and CTA observation configurations.
\end{abstract}

\section{Introduction}

Imaging atmospheric Cherenkov telescopes (IACTs) detect Cherenkov light emitted from air showers of relativistic particles initiated by very-high-energetic (VHE; $\geq 20 \mathrm{GeV}) \gamma$-rays of astrophysical origin. The energy of primary particle is reconstructed by comparing the intensity of the air shower image, related to the flux of Cherenkov photons, with the reference Monte Carlo (MC) shower simulations. The development of the air shower and the emission of Cherenkov light are affected by the molecular density profile of the Earth's atmosphere along the shower direction $[1,2]$. Cherenkov photons can be further attenuated in the air through the absorption and scattering on molecules, aerosols and clouds $[3,4]$. These atmospheric effects contribute to the uncertainty on the reconstruction of primary particles' energies and the effective area of the instrument.

The Cherenkov Telescope Array (CTA) is the future VHE $\gamma$-ray observatory which will improve on the current generation of IACTs in terms of energy coverage, flux sensitivity, angular and energy resolution [5]. To achieve its goals, the CTA aims to restrict the systematic uncertainties due to the atmospheric phenomena through a detailed calibration strategy $[3,4,6]$. Among the foreseen calibration approaches is the method of the Cherenkov Transparency Coefficient (CTC) for the monitoring of the atmospheric transparency to Cherenkov light, $T \propto \exp (-\tau)$, where $\tau$ is the aerosol optical depth.

The CTC was developed within the phase I of the H.E.S.S. experiment with an accuracy of $9 \%$ for the cloudless time periods [7]. The method estimates the atmospheric transparency using the numbers of registered events which are dominated by the background of air

\footnotetext{
*e-mail: stefanik@ipnp.troja.mff.cuni.cz

**Full consortium author list at http://cta-observatory.org
}

showers initiated by charged cosmic ray particles. Although successfully applied for a few telescopes of identical design, the CTC cannot be straightforwardly applied in the CTA utilizing tens of IACTs of different specifications.

Continuing with our preliminary work [8], we discuss the extension of the CTC necessary to meet the CTA performance requirements in Section 2. In Section 3, we demonstrate the sensitivity of our method to the changes in the aerosol concentrations using MC simulations of air showers and the candidate layout of the CTA at its Northern site (CTA-N). In addition, we investigate the impact of different molecular density profiles on the CTC. The conclusions are given in Section 4.

\section{Extension of the Cherenkov Transparency Coefficient}

Building upon the concept of the CTC [7], we consider the minimum detectable energy (trigger energy threshold, $\left.E_{\mathrm{th}}\right)$ of an IACT to be sensitive to the changes in the atmospheric transparency to Cherenkov light, $E_{\mathrm{th}} \propto T^{-1}$. The number of events detected by a telescope above the energy threshold per unit time (trigger rate, $R$ ) is approximately given by $R \propto E_{\text {th }}^{-\gamma} \propto T^{\gamma}$ [7], where $\gamma=\Gamma-1$ with $\Gamma$ being the spectral index of the differential flux of primary particles initiating the air showers. In the first order approach, charged cosmic rays (mainly protons) constitute the majority of particles detected by IACTs. Thus, we assume the value of $\Gamma=2.7$ following from the spectrum of cosmic rays [9].

The atmospheric transparency can be estimated through the comparison of the experimental trigger rate with its estimate obtained from MC simulations for the given observation conditions and reference atmosphere. 


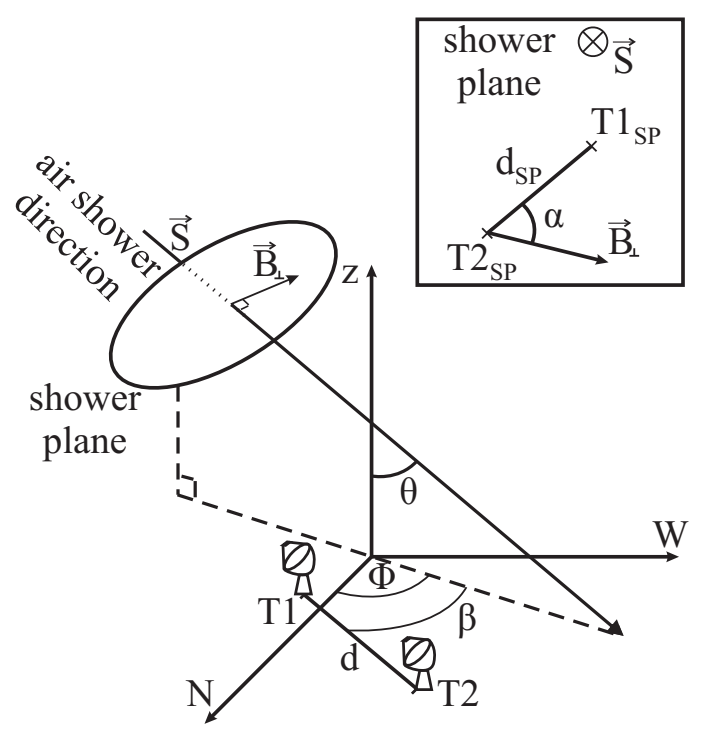

Figure 1. Definitions used in this study. $\beta$ is the angle between the horizontal projection of the air shower direction $(\phi, \theta)$ and the line joining a pair of telescopes (T1, T2) at a distance $d$. Projections from the ground plane into the shower plane orthogonal to the shower direction are labelled with SP. $\alpha$ is the angle between the component of the geomagnetic field transverse to the shower direction $\left(\vec{B}_{\perp}\right)$ and the projection of the line joining T1 and T2.

We define the extended CTC as

$$
\hat{T}=\frac{1}{P} \sum_{\substack{i=1 \\ i<j}}^{N} \hat{T}_{i j}=\frac{1}{P \cdot \mathcal{K}} \sum_{\substack{i=1 \\ i<j}}^{N}\left(\frac{R_{i j}}{\varepsilon_{i} \cdot \varepsilon_{j} \cdot \hat{R}_{i j}^{0}}\right)^{\frac{1}{\gamma}},
$$

where $R_{i j}$ is the experimental rate of events triggering simultaneously a pair of telescopes of a same type assigned as $i$ and $j, \varepsilon_{i}$ and $\varepsilon_{j}$ are the detection efficiencies of the involved telescopes, $\hat{R}_{i j}^{0}$ is the MC rate estimate for $\varepsilon_{i}=\varepsilon_{j}=1, \mathcal{K}$ is the normalization for the nominal atmospheric conditions, $\hat{T}_{i j}$ is the pairwise atmospheric transparency estimate and $P$ is the number of pairs in the array of $N$ telescopes.

In the following, the terms in Eq. (1) are discussed along with the phenomena affecting the trigger rates:

- Random fluctuations. Accidental triggers or the elevated levels of the night sky background (NSB) in the telescope field of view may cause variations of the trigger rate. Random fluctuations are partially mitigated by the usage of the trigger rates of events recorded by at least two telescopes in coincidence (stereoscopic detection).

- Telescope array layout. The layout of active telescopes affects their individual trigger rates recorded in the stereoscopic configuration. For example, telescopes near the centre of the array trigger on average more often than those at its edge because of the larger number of neighbouring telescopes available for the coincidence detection. The dependence on the array layout is simplified if only trigger rates $R_{i j}$ of events detected by selected pairs of telescopes are considered, where $i \neq j$ assign different telescopes.

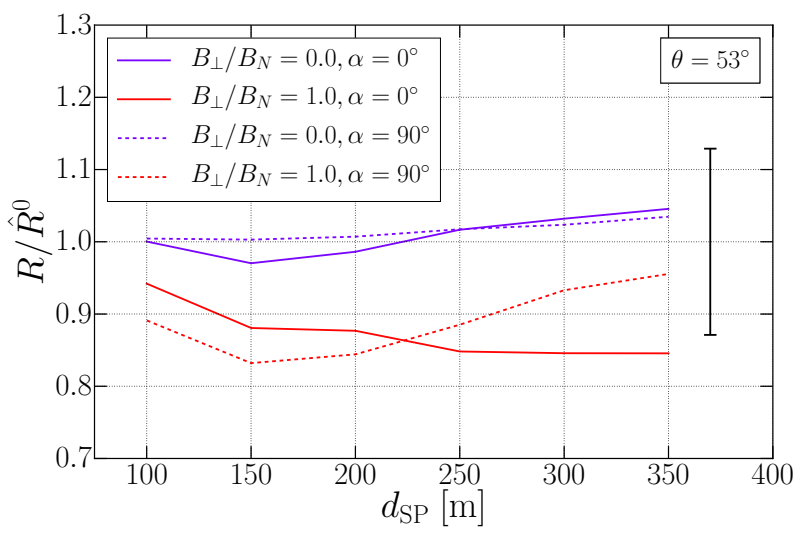

Figure 2. MC trigger rate $(R)$ compared with the fit values $\hat{R}^{0}\left(\theta, d_{\mathrm{SP}}\right)$ [8] for $B \simeq 0$ as a function of the inter-telescope distance in the shower plane $\left(d_{\mathrm{SP}}\right)$. Colours denote the relative size of the geomagnetic field component transverse to the air shower direction $\left(B_{\perp} / B_{\mathrm{N}}\right)$. Solid (dashed) lines correspond to the angle $\alpha=0^{\circ}\left(90^{\circ}\right)$ between $\vec{B}_{\perp}$ and the line joining a pair of telescopes in the shower plane. The error bar illustrates the maximum statistical uncertainty of the data.

- Geometrical configuration. The geometrical definitions used in this study are explained in Figure 1. For air showers developing under increasing zenith angles $(\theta)$, the area on the ground illuminated by Cherenkov light increases while the number of Cherenkov photons per unit area decreases. The pairwise trigger rates are influenced by the combination of these effects, depending also on the inter-telescope distance $(d)$. This dependence is further determined by the alignment angle $(\beta)$ of telescopes with respect to the air shower direction. For a given configuration $(d, \phi, \theta>0)$, the maximum (minimum) of the stereo trigger rate occurs when the line joining a pair of telescopes is parallel (orthogonal) to the shower direction, i.e. $\beta=0^{\circ}\left(90^{\circ}\right)$. The trigger rate is symmetrical with respect to $\beta$ for the intertelescope distance projected in the shower plane, i.e. $d_{\mathrm{SP}}(\theta, \beta)=d \cdot\left[1-\sin ^{2} \theta \cdot \cos ^{2} \beta\right]^{\frac{1}{2}}$. In the absence of the magnetic field and for the nominal telescope optical efficiencies, the pairwise trigger rate can be then approximated by a continuous function $\hat{R}_{i j}^{0}\left(\theta, d_{\mathrm{SP}}\right)$ obtained as a fit of the MC trigger rates, for example (see $[8,10])$.

- Geomagnetic field $(B)$. Due to the influence of the geomagnetic field, the charged particles in the air showers are deflected orthogonally to the component of the magnetic field vector transverse to their momentum vectors $\left(\vec{B}_{\perp}(\phi, \theta)\right)$. The size and shape of the area illuminated by Cherenkov light and the ground density of photons are functions of the size of the transverse component $\left(B_{\perp}\right)$. The energy threshold and, correspondingly, the stereo trigger rate depend on $B_{\perp}$ as well as on the angle $\alpha$ between $\vec{B}_{\perp}$ and the projection of the line joining the telescopes in the shower plane (see Figure 1). In Figure 2, the ratio $R / \hat{R}^{0}\left(\theta, d_{\mathrm{SP}}\right)$ is shown, where $R$ is the MC pairwise trigger rate for $B=B_{\mathrm{N}}, B_{\mathrm{N}}$ is the magnitude of the geomagnetic field at the CTA-N site and $\hat{R}^{0}$ is the trigger rate estimate for $B \approx 0$ obtained from the fit of the geometrical dependencies [8]. The ratio 
is examined for a pair of telescopes separated by various distances $\left(d_{\mathrm{SP}}\right)$ for an example case with $\theta=53^{\circ}$, $B_{\perp} / B_{\mathrm{N}}=0.01\left(\phi=355^{\circ}\right)$ and $1.00\left(119^{\circ}\right)$ and for the angles $\alpha=0^{\circ}$ and $90^{\circ}$. The results suggest that the stereo trigger rate decreases for increasing $B_{\perp}$ and this dependence is modulated by the angle $\alpha$, especially for larger values of $B_{\perp}$ and $d_{\mathrm{SP}}>200 \mathrm{~m}$ (see the red lines in Figure 2). Given the statistical uncertainties of the MC trigger rates (illustrated by the error bar in Figure 2), an increased number of simulations is necessary to estimate the variations of the trigger rate in the possible configurations $\left(B_{\perp}, \alpha, \theta, d_{\mathrm{SP}}\right)$. Restricted by the computational resources, we consider the data in Figure 2 as an estimate of the systematic uncertainties of the CTC due to the geomagnetic field.

- Telescope photon detection efficiency $(\varepsilon)$. The trigger rate of a telescope is affected also by its optical throughput efficiency given by the reflectivity of the optical elements and responses of camera sensors. Assuming the pairwise trigger rate is influenced by the optical efficiencies of all involved telescopes, it can be approximated as $\hat{R}_{i j}\left(\varepsilon_{i}, \varepsilon_{j}\right) \approx \varepsilon_{i} \cdot \varepsilon_{j} \cdot \hat{R}_{i j}^{0}$, where $0 \leq \varepsilon_{i} \leq 1$ refers to the percentage of the nominal optical efficiency of a telescope $i$ and $\hat{R}_{i j}^{0}$ is the trigger rate estimate for $\varepsilon_{i}=\varepsilon_{j}=1$. Note that the optical efficiencies of telescopes have to be estimated in an independent procedure [11-14]. A further influence of the telescope hardware is the possibility of adapting the camera trigger thresholds to the measured rates of individual camera pixels. The implementation of the CTC under these conditions (foreseen for the large-size telescopes in CTA) will be investigated in a future work.

The CTC in Eq. (1) is independent of observational and hardware effects provided the influence of the geomagnetic field in the given observation is small and the telescope optical efficiencies were correctly estimated. Thus, the CTC is sensitive only to the changes in the atmospheric transparency (equivalently, the aerosol optical depth) to Cherenkov photons below the production height of air showers.

\section{Atmospheric calibration using the CTC}

We examine the sensitivity of the extended CTC method to the variations in the aerosol concentrations and molecular density profiles using MC simulations of the protoninitiated air showers observed by the CTA.

The simulations were performed using the CORSIKA [15] and sim_telarray [16] software. The air showers were initiated by protons with energies $0.004-$ $100 \mathrm{TeV}$. We simulated the full candidate CTA-N layout comprising 4 large-size (LST) and 15 medium-size (MST) telescopes with the models characterized in the CTA simulation production [17]. Degradation of the telescope hardware was included by drawing the optical efficiencies $(\varepsilon)$ randomly from the normal distribution $\mathcal{N}(0.7,0.1)$.

The sensitivity of the CTC to different concentrations of aerosols was studied using the tropospheric, desert, navy maritime and no aerosol models created by the

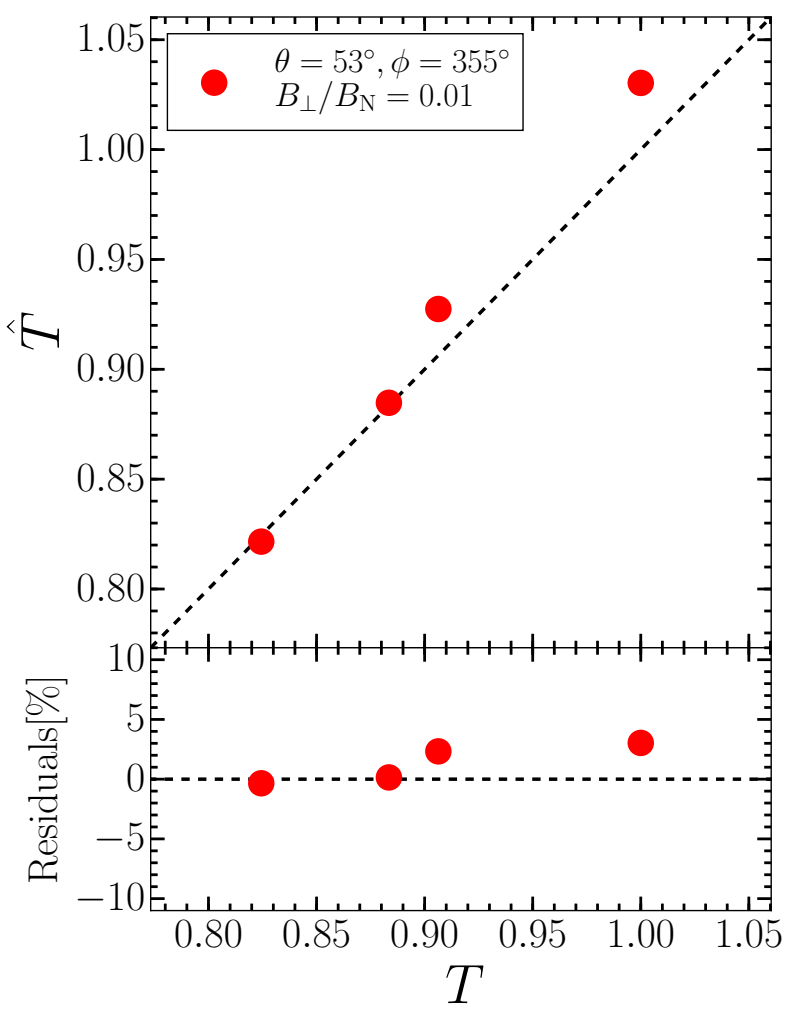

Figure 3. The CTC estimates $(\hat{T})$ compared to the atmospheric transparencies inserted as input in MC simulations $(T)$ for two example configurations (different markers). The residuals are shown below. Dashed lines illustrate the perfect agreement.

MODTRAN program [18]. The atmospheric transparency corresponding to these models $(T \in\{0.82,0.88,0.91,1.0\}$, respectively) was ascertained as the median value of $\exp (-\tau(\lambda)) \cdot R(\lambda) \cdot Q(\lambda)$, where $\tau$ is the optical depth for the altitude of $12 \mathrm{~km}^{1}, R$ is the reflectivity of telescope mirrors, $Q$ is the efficiency of camera photo-sensors and $\lambda$ is the wavelength of incident light. The transparency was normalized to $T=1.0$ for the model without aerosols.

In Figure 3, the CTCs $(\hat{T})$ calculated according to Eq. (1) are compared with the atmospheric transparency assumed in $\mathrm{MC}$ simulations $(T)$ for an example case with $\theta=53^{\circ}, \phi=355^{\circ}$, corresponding to $B_{\perp} / B_{\mathrm{N}}=0.01$. The resolution of the CTC in the shown example is about $3 \%$.

The results in Figure 3 are shown for a single profile of the molecular density representative of the conditions at the CTA-N site ('CTA-N template') [19]. In order to examine the influence of the change in the density on the CTC, we performed simulations with various molecular profiles in the configuration $\theta=53^{\circ}, \phi=355^{\circ}, B_{\perp} / B_{\mathrm{N}}=$ $0.01, T=1.00$. The obtained CTCs are listed in Table 1 for the template profile, the average summer and winter profiles at the CTA-N site and for the 'extreme' profiles assuming the minimum (maximum) of the air density to occur at the altitude of 7 and $14 \mathrm{~km}(5$ and $16 \mathrm{~km})$. All CTC estimates are consistent with the input atmospheric

\footnotetext{
${ }^{1}$ We assume that the atmospheric attenuation of Cherenkov light dominates below the maxima of air showers which typically happen below the altitudes of $\sim 12 \mathrm{~km}$ (for energies of CRs above $10 \mathrm{GeV}$ [1])
} 
Table 1. The CTC calculated from MC data with different molecular density profiles. Statistical and systematic uncertainties of the estimates are $\sim 14 \%$ and $5 \%$, respectively.

\begin{tabular}{ll}
\hline Profile & $\hat{T}$ \\
\hline CTA-N template & 1.03 \\
Average summer & 0.99 \\
Average winter & 1.01 \\
Density minimum at $14 \mathrm{~km}$ & 0.99 \\
Density maximum at $16 \mathrm{~km}$ & 1.00 \\
Density minimum at $7 \mathrm{~km}$ & 1.00 \\
Density maximum at $5 \mathrm{~km}$ & 1.02 \\
\hline
\end{tabular}

transparency $(T=1.00)$ within the estimated resolution. Thus, the CTC provides a reliable measure of the aerosol optical depth while remaining insensitive to the changes in the molecular density profiles.

It is worth noting that the trigger rate estimates $\hat{R}_{i j}$ input in Eq. (1) were obtained separately for the LST and MST pairs as a fit of the MC pairwise trigger rates for different $\left(\theta, d_{\mathrm{SP}}\right)$. For details of this fit see $[8,10]$.

The CTC was calculated assuming the telescope optical efficiencies which were used as input for MC simulations. In a more realistic scenario, the optical throughput will be estimated by an independent method with a certain precision $(\sim 4 \%$ for the calibration using muon ring images [11], for example) which will contribute to the uncertainty of the CTC estimates.

Statistical uncertainties of the MC trigger rates are 10$20 \%$, caused by the small trigger efficiency to proton air showers in some bins of energy and impact parameter. We note that the uncertainty of the CTA experimental trigger rate will be $<1 \%$ for the typical observation runs.

\section{Conclusions}

We presented the extended CTC method for the monitoring of the atmospheric transparency to Cherenkov light in the extensive arrays of IACTs. The method relies on the usage of the pairwise trigger rates of telescopes, carefully accounted for their observational and instrumental dependencies. Calculated using telescope data without the need for special devices or data acquisition, the CTC approach does not interfere with the operation of the instruments on site. Complementary to other atmospheric monitoring devices, the CTC is a robust estimate of the atmospheric transparency advantageously determined in the field of view and time range of the CTA observations.

In the particular case of the Northern array of the CTA observatory, we showed in an example MC simulation that the resolution of the method is $3 \%$ for different aerosol concentrations in the air. Given the accuracy of the method, variations in the molecular density profiles cannot be recognized by the CTC.

The future steps will include the implementation of the CTC for the CTA small-size telescopes (SSTs), the appli- cation under different levels of the NSB and adaptable trigger thresholds of the LSTs. Eventually, the CTC will be cross-checked with other atmospheric calibration methods after the deployment of first telescopes on the CTA sites.

This work was conducted in the context of the CTA Central Calibration Facilities Work Package. We gratefully acknowledge financial support from the agencies and organizations listed here: http://www.cta-observatory.org/consortium_acknowledgments. We are also grateful for the support by the grants LTT17006 and LM2015046 of the Ministry of Education, Youth and Sports of the Czech Republic.

\section{References}

[1] K. Bernlöhr, Astroparticle Physics 12, 255 (2000)

[2] M. Vrastil, European Physical Journal Web of Conferences 144, p. 01014 (2017)

[3] J. Ebr, T. Bulik, L. Font et al., Proceedings of the 35th ICRC, Busan (2017)

[4] M. Gaug, European Physical Journal Web of Conferences 144, p. 01003 (2017)

[5] B.S. Acharya, M. Actis, T. Aghajani et al., Astroparticle Physics 43, 3 (2013)

[6] M. Gaug, D. Berge, M. Daniel et al., Proceedings of SPIE 9149, p. 914919 (2014)

[7] J. Hahn, R. de los Reyes, K. Bernlöhr et al., Astroparticle Physics 54, 25 (2014)

[8] S. Stefanik, R. de los Reyes, D. Nosek, Proceedings of the 35th ICRC, Busan (2017)

[9] T. Sanuki, M. Motoki, H. Matsumoto et al., ApJ 545, 1135 (2000)

[10] S. Stefanik, D. Nosek, R. de los Reyes et al., Atmospheric monitoring and inter-calibration of the telescope optical throughput efficiencies using the trigger rates of the Cherenkov Telescope Array, submitted for publication

[11] M.C. Maccarone, D. Parsons, M. Gaug et al., Proceedings of the 35th ICRC, Busan (2017)

[12] M. Gaug, S. Fegan, A. Mitchell et al., Using muon rings for the optical throughput calibration of the Cherenkov Telescope Array - Part I, submitted for publication

[13] A.M. Brown, Astroparticle Physics 97, 69 (2018)

[14] A. Segreto, M.C. Maccarone, O. Catalano et al., Proceedings of SPIE 9149, p. 9906 (2016)

[15] D. Heck, J. Knapp, J.N. Capdevielle, G. Schatz, T. Thouw, A Monte Carlo code to simulate extensive air showers (Forschungszentrum Karlsruhe GmbH, Karlsruhe, Germany, 1998)

[16] K. Bernlöhr, Astroparticle Physics 30, 149 (2008)

[17] G. Maier, L. Arrabito, K. Bernlöhr et al., Proceedings of the 35th ICRC, Busan (2017)

[18] A. Berk, P. Conforti, R. Kennett et al., Proceedings of SPIE 9088 (2014)

[19] P. Munar-Adrover, M. Gaug, Studying molecular profiles above the Cherenkov Telescope Array sites, these proceedings 